\title{
Quantum limitations on superluminal propagation
}

\author{
Yakir Aharonov $^{(a)}$, Benni Reznik ${ }^{(b)}$ and Ady Stern ${ }^{(c)}$ \\ (a) School of Physics and Astronomy, Tel Aviv University, Tel Aviv 69978, Israel, and \\ Department of Physics, University of South Carolina, Columbia, SC 29208. \\ (b) Theoretical Division, T-6, MS B288, Los Alamos National Laboratory, Los Alamos, NM, 87545 \\ (c) Department of Condensed Matter Physics, Weizmann Institute of Science, Rehovot 76100, Israel
}

Unstable systems such as media with inverted atomic population have been shown to allow the propagation of analytic wavepackets with group velocity faster than that of light, without violating causality. We illuminate the important role played by unstable modes in this propagation, and show that the quantum fluctuations of these modes, and their unitary time evolution, impose severe restrictions on the observation of superluminal phenomena.

A famous consequence of Einstein's special theory of relativity is the principle of causality: signals cannot travel faster than light. Nevertheless, it is known for some time, that under certain conditions the group velocity of an electromagnetic wavepacket can be arbitrarily large and its energy positive, but yet no conflict arises with causality: the signal velocity remains always smaller than the velocity of light in vacuum. At least two classes of such models allowing "causal" superluminal behavior, are known: the first is closely related to tunneling phenomena, the second arises for unstable systems of atoms under an inverted population condition [1].

While in the first case the "superluminal" transmission of waves or particles through a barrier is exponentially suppressed, the second case shows much more dramatic superluminal-like effects. Wavepackets travel with a superluminal group velocity for unlimited distance, with negligible attenuation and dispersion. The second phenomenon arises due to instabilities in the initial state of the radiating system, for example, a scalar field initially in a "false vacuum" state [2]. Recently, an optical experiment studying superluminal group velocities for waves in a medium with inverted population was suggested [3 5]. While the discussion was limited so far to the semi-classical limit, it was further suggested [5], that with a full fledged quantum-mechanical treatment one would possibly obtain stable tachyonic-like quasiparticle excitations in the inverted medium.

In this Letter we examine classical and quantum aspects of superluminal group velocities in unstable systems. Classically, a peak of an analytic wavepacket may travel from point $A$ to point $B$ faster than the speed of light, since its shape at $B$ can be fully reconstructed from the part of the wave packet which is causally connected to the point $B$. We show that quantum mechanically, analyticity of the wave packet is not the only condition needed for such a reconstruction. Rather, the part of the wave packet which is causally connected to the point $B$ must contain many photons or large enough energy in the unstable modes. This condition strongly suppresses superluminal effects in the limit that the system contains only a few photons. The exponential suppression of superluminal effects characterizes both the tunneling phenomena and the present case of unstable systems.

To begin with we introduce a simple model which classically exhibits tachyonlike motion. Consider a real scalar field $\varphi(x, t)$ in one spatial dimension, under the Hamiltonian

$$
H=\frac{1}{2} \int d x\left(\pi^{2}+\left(\partial_{x} \varphi\right)^{2}+\frac{2 m^{2}}{\lambda} \cos \sqrt{\lambda} \varphi\right)
$$

Here, $\pi(x, t)=\dot{\varphi}(x, t)$ is the field conjugate to $\varphi(x, t)$, $\lambda>0$ and the speed of light is put equal to one. This Hamiltonian describes the continuum limit of coupled pendula. In the stable ground state $\varphi(x)=\pi$. We will be interested, however, in the dynamics of $\varphi$ near the metastable state $\varphi(x)=0$. For any finite time interval of interest, we may take $\lambda$ sufficiently small such that the potential term may be expanded, $\cos \sqrt{\lambda} \varphi \approx 1-\frac{\lambda}{2} \varphi^{2}$. From now on we restrict ourselves to this expansion. Then, the equation of motion for $\varphi$ becomes,

$$
\square \varphi-m^{2} \varphi=0
$$

In term of the eigenmodes the solution is

$$
\begin{aligned}
& \varphi(x, t)=\frac{1}{\sqrt{2 \pi}} \int d k e^{i k x} \varphi_{k}(t) \\
& \pi(x, t)=\frac{1}{\sqrt{2 \pi}} \int d k e^{i k x} \pi_{k}(t)
\end{aligned}
$$

where reality conditions imply $\varphi_{-k}=\varphi_{k}^{\dagger}, \quad \pi_{-k}=\pi_{k}^{\dagger}$. The time evolution of the modes is given by

$$
\begin{aligned}
& \varphi_{k}(t)=\varphi_{k 0} \cos \omega_{k} t+\frac{\pi_{0 k}}{\omega_{k}} \sin \omega_{k} t \\
& \pi_{k}(t)=\pi_{0 k} \cos \omega_{k} t-\omega_{k} \varphi_{0 k} \sin \omega_{k} t
\end{aligned}
$$

where

$$
\omega_{k}^{2}=k^{2}-m^{2}
$$

Notice that for $|k|>m, \omega_{k}$ is real, and $\varphi_{k}, \pi_{k}$ oscillate in time. In the range $|k|<m \omega_{k}$ is imaginary, and 
$\varphi_{k}(t)$ and $\pi_{k}(t)$ are exponentially diverging. The latter modes are analogous to spontaneous emission in the optical model of an inverted medium of two-level systems. We will henceforth refer to the free oscillatory modes and diverging modes as the "normal" and "unstable" modes, respectively. It is only near the point $\varphi \sim \pi \sim 0$ that these modes exist as linearly independent solutions. As $\varphi$ grows sufficiently, the instability is damped by the nonlinear $\lambda$-term in (1D), but for the time of interest to us, the latter can be neglected.

We now turn to examine several features of the classical equation of motion (2). First, we examine the propagation of a wavepacket given by

$$
\varphi(x, t)=\int_{|k|>m} d k\left(g_{0}(k) e^{i k x-i \omega_{k} t}+h . c .\right)
$$

and $\pi(x, t)=\partial_{t} \varphi(x, t)$. We take $g_{0}(k)$ to be non zero only in the range of normal modes $m<|k|<k_{\max }$, and centered around $k=k_{0}$, with a width $\Delta k \ll k_{0}-m$. The spatial width of this wavepacket is $1 / \Delta k$, and we assume that at $t=0$ it is spatially centered very far to the left of the origin, at $X_{0} \ll 0$.

This wavepacket propagates with a group velocity, $v_{g}$, and a phase velocity, $v_{p}$, given by

$$
v_{g}=\frac{1}{v_{p}}=\frac{k_{0}}{\sqrt{k_{0}^{2}-m^{2}}}
$$

Since $v_{g}>1$, the motion of the center of the wave packet (group velocity) is superluminal (tachyonlike), while the phase velocity is always subluminal. As long as the dispersion is negligible, $\varphi(x, t)$ is (up to a time dependent phase factor $)$ just $\varphi\left(x-v_{g} t, 0\right)$, i.e., the initial wavepacket moving at velocity $v_{g}$.

Second, we note that despite the superluminal group velocity, causality is maintained [2.,6]. This is best seen through the Green's functions of Eq. (2). In terms of the homogeneous Green functions: $\varphi(x, t)=\int d x^{\prime}[G(x-$ $\left.\left.x^{\prime}, t\right) \varphi_{0}\left(x^{\prime}\right)+\tilde{G}\left(x-x^{\prime}, t\right) \pi_{0}\left(x^{\prime}\right)\right]$. However, $\tilde{G}$ and $G$ vanish outside the light cone, i.e., for $x-x^{\prime}>t$, therefore the value of $\varphi$ at point $x$ at time $t$ is fully determined by its value at points $x^{\prime}$ that are causally connected to $x$.

For further insight into this point, it is instructive to examine what happens if the initial wavepacket is truncated at $x=0$, i.e., $\varphi_{0}(x)$ and $\pi_{0}(x)$ are replaced by $\varphi_{0}^{T}=\tilde{\theta}(x) \varphi_{0}(x)$ and $\pi_{0}^{T}=\tilde{\theta}(x) \pi_{0}(x)$, respectively. Here $\tilde{\theta}$ is a smoothed step function. The length scale over which it is smoothed is assumed smaller than all other length scales in the problem, and is kept finite just to avoid an infinite $\partial_{x} \varphi$. Note that since $\varphi_{0}(x)$ is centered at $X_{0} \ll 0$, the truncated wavepacket, $\varphi_{0}^{T}(x)$ constitutes only the tail of the original one, and the energy stored in the truncated wavepacket is just a small fraction of that stored in the original one. The momentum representation of the truncated wavepacket $\varphi_{0}^{T}(x)$ is
$g_{0}^{T}(k)=\int d k^{\prime} \frac{1}{k-k^{\prime}+i \eta} g_{c}\left(k^{\prime}\right)$, with $\eta$ an infinitesimal number. The smoothing of the step function is accounted for by introducing an upper cut-off to the $k$ integral. While the original wavepacket was solely composed of normal modes, the truncated one includes also unstable components.

The time evolution of the truncated wavepacket can be calculated using the exact expression for the Green function [2,3,6]. There are three regimes: (a.) For points $x<-t$, the causality of the Green function dictates that the value of $\varphi_{T}(x, t)$ is not affected by the existence of the truncated wavepacket at $x>0$. Thus, the field is zero in this range. (b.) For $-t<x<t$, however, the time evolution of the truncated wavepacket is very different from that of the original one: it is exponentially growing due to the contributions of unstable components. Since the amplitude of the normal modes oscillates in time while the amplitude of the unstable modes grows exponentially, for times $t \gg 1 / m$ the wavepacket $\varphi^{T}(x, t)$ is predominantly composed of unstable modes of very small wavevectors, with an exponentially small contribution of normal modes. (c.) Finally, for points $x>t$, the causality of the Green function dictates that the value of $\varphi_{T}(x, t)$ does not depend on whether or not it is truncated at the origin at $t=0$. It is given, up to a time dependent phase factor, by $\varphi\left(x-v_{g} t, 0\right)$. The propagation of the superluminal peak is not affected by the truncation, and, for long enough times, one finds at $X_{0}+v_{g} t$ a wavepacket of width $1 / \Delta k$, in which the field $\varphi$ oscillates with a characteristic wavevector of $k_{0}$. Thus, at long times the field $\varphi^{T}(x, t)$ shows an interesting behavior: it is predominantly composed of very small wavevectors $|k|<m$ with just an exponentially small contribution of wavevectors $|k|>m$. However, in a region of spatial width $1 / \Delta k$ around $X_{0}+v_{g}$ t it oscillates at the large wavevector $k_{0}>m$. The region $1 / \Delta k$ can be made arbitrarily large, if $t$ is taken long enough. These shortwavelength oscillations of a long-wavelength wavepacket are very similar to the superfast Fourier oscillations discussed in [7] 9$]$.

The above discussion demonstrates that in order to maintain consistency with causality, the mechanism which gives rise to superluminal group velocities has to rely on the local information stored in the tail. As long as the true information that the wave has been truncated has not arrived, the local amplification extrapolates the peak as if this truncation does not exist. Classically, the possibility of extrapolating the full wave from an infinitesimally small tail is, although surprising, possible. As discussed in [6], for an analytic function, the wave can be reconstructed by means of a local Taylor expansion, and the tachyonlike propagation can be viewed as an analytic continuation of the tail.

As we now show, the extrapolation of the full propagated wavepacket from the truncated tail is made possible by the unstable modes, and this is true independent 
of the details of the model. Since $g_{0}^{T}(k)$ is the Fourier transform of a small tail, it is very small. The propagated wavepacket, after truncation, is

$$
\varphi^{T}(x, t)=\int d k\left(e^{i k x-i \omega_{k} t} g_{0}^{T}(k)+h . c .\right)
$$

However while on the right hand side, the amplitudes $g_{0}^{T}(k)$, originating from the truncated tail, are very small, on the left side the magnitude of the tachyonic peak is not small, since the peak is fully reconstructed from the tail. Since the amplitude of the stable modes, $\varphi_{T}(k)$, for $|k|>m$, is constant in time, this amplification can arise only from the contribution of unstable modes growing exponentially with time. It is then essential to have unstable modes for a full reconstruction of a superluminal peak from a truncated tail.

We now proceed to the quantum mechanical analog of the classical superluminal system. Can a similar mechanism of local amplification give rise to superluminal group velocities? If so, it is conceivable that by means of a process, analogous to the classical analytical continuation, this local information may be used to reconstruct the wavepacket's peak.

The causality of the Hamiltonian (1) is manifested in the quantum case by the statement that causally disconnected local observables commute. Again, this causality motivates us to study the quantum analog of the classical truncated wavepacket. In this study, detailed below, we define an analog of a "false vacuum" state for this model, on top of which we define a quantum state describing a truncated wavepacket. Then, we show that if the truncated wavepacket is too small (in a sense to be explained below), the initial quantum state describing it is not orthogonal to the false vacuum state. In that case, the vacuum quantum fluctuations in the fields $\varphi, \pi$ are, at $t=0$, larger than the amplitude of the wavepacket, and one cannot distinguish between the false vacuum state and the wavepacket state. As we saw above, classically the small truncated wavepacket becomes exponentially large in time, and one may naively hope that quantum mechanically it eventually becomes larger than the vacuum fluctuations. However, this cannot be the case, due to the unitary quantum mechanical time evolution. By the principle of unitarity, the scalar product of two states, here the false vacuum and truncated wavepacket states, is constant in time. Thus, if at $t=0$ the truncated wavepacket is not orthogonal to the false vacuum, it cannot be distinguished from it at all later times. The exponential growth of the tail is masked by an exponential growth in the quantum fluctuations.

Turning to a more concrete discussion, we promote the functions $\varphi(x, t), \pi(x, t)$ to quantum operators. From the standard canonical commutation relations for the fields, $\left[\varphi(x, t), \pi\left(x^{\prime}, t\right)\right]=i \delta\left(x-x^{\prime}\right)$, it follows as usual that for the normal modes: $\left[\varphi_{k}, \pi_{k^{\prime}}^{\dagger}\right]=\left[\varphi_{k}^{\dagger}, \pi_{k^{\prime}}\right]=i \delta\left(k-k^{\prime}\right)$.
However, for the unstable modes the the system does not admit the usual Fock space structure with an ordinary particle interpretation. For these modes $(|k|<m)$, the Hamiltonian, $\sum_{k} \pi_{k}^{\dagger} \pi_{k}-\left(m^{2}-k^{2}\right) \varphi_{k}^{\dagger} \varphi_{k}$, is unbounded from bellow, and has a continuous and unbounded spectrum of energies for each wave number $k$. Consequently, these modes cannot be put in a ground state, and the fluctuations in the fields $\varphi_{k}, \pi_{k}$ inevitably grow exponentially with time.

For the normal modes $|k|>m$ the vacuum state is obviously the ground state of an harmonic oscillator of frequency $\sqrt{k^{2}-m^{2}}$. The situation is less obvious for the unstable modes. On one hand, we would like the quantum fluctuations in the fields $\varphi_{k}, \pi_{k}$ to be as small as possible, but, on the other hand, stationary states of the unstable modes necessarily have infinite fluctuations. Any state of finite fluctuations is non-stationary, with the fluctuations growing exponentially in time. Thus, we choose the initial state of the unstable modes to be a non-stationary one, in which the exponential growth of the field fluctuations is slowest. This state is the ground state of an harmonic oscillator of frequency $\sqrt{m^{2}-k^{2}}$. The vacuum state of the entire system is then a direct product of the vacuum state for each mode $k$.

Next, we should find the quantum analog of the wave packet we analyzed in the classical case. Again, causality dictates that a measurement of the field $\varphi$ at a given point cannot be affected by its values in causally disconnected points. Thus, to analyze the quantum analog of the classical super-luminal propagation, we need to construct the quantum analog of the truncated wavepacket $\varphi_{0}^{T}(x)$. We do so by shifting each mode $\varphi_{k}$ from its vacuum state, in which $\left\langle\varphi_{k}\right\rangle=0$, to a coherent state, in which $\left\langle\varphi_{k}\right\rangle=g_{0}^{T}(k)$ :

$$
\left|\Psi_{0}\right\rangle=\exp \left[i \sum_{k} g_{0}^{T}(k) \hat{\pi}_{k}^{\dagger}\right]|\mathrm{vac}\rangle
$$

where $|v a c\rangle$ is the vacuum state. Classically, as we saw above, the truncated wavepacket included all the information needed to reconstruct the superluminal propagation of $\varphi_{0}(x)$. Quantum mechanically, however, this is true only if the state (10) is orthogonal to the vacuum state. If that is not the case, one cannot distinguish between the time evolution of the vacuum state and that of (10).

The scalar product $\left\langle\mathrm{vac} \mid \Psi_{0}\right\rangle$ can be easily calculated. It is,

$$
\left\langle\operatorname{vac} \mid \Psi_{0}\right\rangle=\exp -\frac{1}{2 \hbar} \int d k\left|\omega_{k}\right|\left(g_{0}^{T}(k)\right)^{2}
$$

It is useful to distinguish between the normal $(|k|>m)$ and unstable $(|k|<m)$ contributions to the $k$-integral in (11). The physical interpretation of the normal contributions is rather clear: $g_{0}^{T}(k)$ is the amplitude of the 
oscillations of the mode $k$, and $\omega(k) \mid\left(g_{0}^{T}(k)\right)^{2}$ is the average number of photons in that mode. The contribution of the unstable modes cannot be discussed in terms of the photons. For these modes, the integrand $\frac{1}{\hbar}|\omega(k)|\left(g_{0}^{T}(k)\right)^{2}$ is just the ratio of the kinetic energy stored in the $k$-mode to $\hbar \omega(k)$. The quantum state containing the wavepacket, $\left|\Psi_{0}\right\rangle$, is then orthogonal to the vacuum state only if the truncated wavepacket contains many photons ( $\gg 1$ ) of the normal modes, or large enough energy in the unstable modes. This poses a new condition, which did not exist in the classical theory, for the reconstruction of a tachyonic peak from a truncated tail: the truncated tail cannot be too small. If it is too small, the initial fluctuations in the field $\varphi$, which grow exponentially with time, overcome the reconstructed peak.

To exemplify the last point, let us evaluate the fluctuations of a local observable. The field at a point has singular fluctuations, and one needs to consider smeared operators like: $\bar{\varphi}_{(L, t)} \equiv \int f\left(x^{\prime}\right) \varphi\left(L-x^{\prime}, t\right) d x^{\prime}$. where $f\left(x^{\prime}\right)$, is non-vanishing only in the spacetime volume $\Delta L$ around the point $L$, and is normalized to $\int f d x=1$. Since $\left\langle\Psi_{0}|\bar{\varphi}| \Psi_{0}\right\rangle$ is identical to the classically smeared field, the fluctuations are dominated by $\left\langle\Psi_{0}\left|\bar{\varphi}^{2}\right| \Psi_{0}\right\rangle=$ $\left\langle\bar{\varphi}^{2}\right\rangle_{N}+\left\langle\bar{\varphi}^{2}\right\rangle_{U}$ where the subscripts $N$ and $U$, stand for the contributions of the normal and unstable modes. The contribution of the normal modes is always finite. On the other hand, the unstable band yield an exponentially growing fluctuation:

$$
\left\langle\bar{\varphi}^{2}\right\rangle_{U} \approx \hbar I_{0}[2 m T] \stackrel{T \gg 1 / m}{\rightarrow} \hbar \frac{e^{2 m T}}{\sqrt{4 \pi m T}}
$$

where $I_{0}$ is zeroth order modified Bessel function. For the observation of superluminal propagation two conditions have to be satisfied: First $v_{g} T_{o b s} \gg \frac{1}{\Delta k}$ (where $T_{o b s}$ is the time at which the wavepacket is observed), i.e., the point of observation should be far outside the initial spread of the wavepacket. Second, we must distinguish between superluminal propagation and propagation at the speed of light. This leads to: $\left(v_{g}-1\right) T_{o b s} \gg \frac{1}{\Delta k}$. These conditions require that $T_{\min } m \gg 1$. Eq. (12) thus implies that for the signal amplitude to be larger than the amplitude of the fluctuations at the observation time, the signal amplitude should ne exponentially large.

One may still question the generality of our result. For instance we have assumed that initialy the unstable modes are in a direct product state. Can a different initial state be made orthogonal to the vacuum more easily? We now argue that the obstacles pointed above in the way to realizing quantum mechnical superluminal group velocities cannot be circumvented. Consider three initial classical tachyonlike wavepackets, denoted by $R, L$ and $D$, with intial field and momenta configurations $\left(\varphi_{R}, \pi_{R}\right),\left(\varphi_{L}=\varphi_{R}, \pi_{L}=-\pi_{R}\right)$, and $\left(\varphi_{D}=-\varphi_{R}, \pi_{D}=\pi_{R}\right) . \quad R$ and $L$ represent right and left moving wave packets with the same initial $\varphi, D$ is right moving with negative $\varphi$. Suppose that initially the wavepackets are localized around $X_{0} \ll 0$. Then after some time, $R$ and $D$ form peaks (of opposite sign) at $X \gg 0$ while $L$ leaves at $X \gg 0$ only a small tail. Again, we consider the quantum states analogous to the classical truncated tails of the three wavepackets, denoted by $R^{T}, L^{T}, D^{T}$, where the truncation is done at $X=0$. Classically, all three wavepackets can be reconstructed from their tails. Quantum mechanically, however, it is not possible for all three quantum states to be mutually orthogonal: $R^{T}$ and $L^{T}$ are orthogonal if the uncertainty in the momentum in each state is smaller than the difference between the momentum expectation value of the two states: $\Delta \pi_{(L, R)}^{T} \ll\left\langle\pi_{R}^{T}\right\rangle-\left\langle\pi_{L}^{T}\right\rangle$. Similarly, $R$ and $D$ are orthogonal if $\Delta \varphi_{(R, D)}^{T} \ll\left\langle\varphi_{L}^{T}\right\rangle-\left\langle\varphi_{R}^{T}\right\rangle$. However, at the tail, the right hand side of these two inequalities can be made arbitrarily small, while the left hand side cannot be made arbitrarily small, due to the uncertainty principle. Thus, if the tails are small enough, the classically distinguishable wavepackets $R, L$ and $D$ cannot be mapped into three mutually orthogonal quantum states.

In conclusion, we have shown that classical superluminal - like effects become incompatible with unitarity in the quantum mechanical limit, and are strongly suppressed. Our argument strongly questions the possiblity that these systems may have tachyon-like quasiparticle excitations made of a small number of photons [5].

Acknowledgment Y. A. acknowledges the support of the Basic Research Foundation, grant 614/95, administered by the Israel Academy of Sciences and Humanities. A.S. acknowledges support of the US-Israel Bi-national Science Foundation (95/250-1), the Minerva foundation and the V. Ehrlich career development chair. We thank I. Ussishkin for constructive discussions.

[1] For a recent review see: R. Y. Chiao and A. M. Steinberg, Progress in optics 37, ed. E. Wolf (1998).

[2] Y. Aharonov, A. Komar and L. Susskind, Phys. Rev. 182, 1400 (1969).

[3] R. Y. Chiao, Phys. Rev. A. 48, R34, (1993).

[4] R. Y. Chiao and J. Boyce, Phys. Rev. Lett. 73, 3383 (1994).

[5] R. Y. Chiao, A. K. Kozhekin and G. Kurizki, Phys. Rev. Lett. 77, 1254 (1996).

[6] G. Diener, Phys. Lett. A223, 327 (1996)

[7] Y. Aharonov, D. Z. Albert, and L. Vaidman, Phys. Rev. Lett., 14, 1351 (1988).

[8] M. V. Berry, J. Phys. A: Math. Gen., 27, L391 (1994).

[9] B. Reznik, Phys. Rev. D55, 2152-2158, (1997). 